\title{
Some Inferences on Skew-t Distribution of 2 Degrees of Freedom
}

\author{
Mohammad Ahsanullah \\ Department of Management Sciences \\ Rider University, Lawrenceville, New Jersey, USA \\ Valery B. Nevzorov \\ Department of Mathematics and Mechanics \\ St. Petersburg State University, St. Petersburgh, Russia
}

Received 15 January 2017

Accepted 15 August 2017

\begin{abstract}
Understanding the properties of Skew distributions is important in many statistical applications. The Student-t distribution is widely used in statistics. Skew-t distribution is a useful model to describe data with heavy tails. In this paper several distributional properties of Skew-t distribution are given. Based on the distributional properties some characterizations of the Skew-t distribution are given.
\end{abstract}

Keywords: Skew-t Distribution; Percentile Points; Characterizations.

\section{Introduction}

A random variable $X$ is said to have the skew-t distribution if its probability density function (pdf) is of the form $f_{S T}(x)=2 g(x) G(\alpha x)$ where $g(x)$ and $G(x)$ are respectively the pdf and the cumulative distribution function (cdf). The pdf $f_{S T}(x, \alpha)$ of skew-t distribution with degrees of freedom $v=2$ and with $\alpha$ as a real number is given below.

$$
f_{S T}(x, \alpha)=\frac{1}{\left(2+x^{2}\right)^{3 / 2}}\left(1+\frac{\alpha x}{\sqrt{2+\alpha^{2} x^{2}}}\right), \quad-\infty<x<\infty .
$$

The Skew-t distribution with 2 degrees of freedom was proposed by Azzalini [8] as a useful model to describe data with heavy tails. Jamalizadeh et al. [9] have shown a recurrence relation of the skew-t distribution of $v+$ 1 degrees of freedom with the Student t-distribution of $v(\geq 2)$ degrees of freedom. For $v=2$, the cdf $F_{S T}(x, \alpha)$ of the skew-t is as follows.

$$
F_{S T}(x, \alpha)=\frac{1}{2}-\frac{1}{\pi} \arctan \alpha+\frac{x}{\sqrt{2+x^{2}}}\left(\frac{1}{2}+\frac{1}{\pi} \arctan \left(\frac{\alpha x}{\sqrt{2+x^{2}}}\right)\right), \quad-\infty<x<\infty .
$$

The corresponding pdf is

$$
f_{S T}(x, \alpha)=\frac{1}{\left(x^{2}+2\right)^{3}}\left(1+\frac{2}{\pi} \arctan \frac{\alpha x}{\sqrt{x^{2}+2}}+\frac{2}{\pi} \frac{\alpha x \sqrt{x^{2}+2}}{\left(1+\alpha^{2}\right) x^{2}+2}\right),
$$

The pdfs given at (1) and (3) are close to each other but not exactly the same and they coincide at $\alpha=0$. In this paper we will consider some distributional properties and characterizations of the skew-t distribution with the pdf as given in (3). 


\section{Main Results}

There are similarities of $f_{S T}(x,-\alpha)$ and $f_{S T}(x, \alpha)$. See figure 1 for $\alpha=-2$ and 2 .

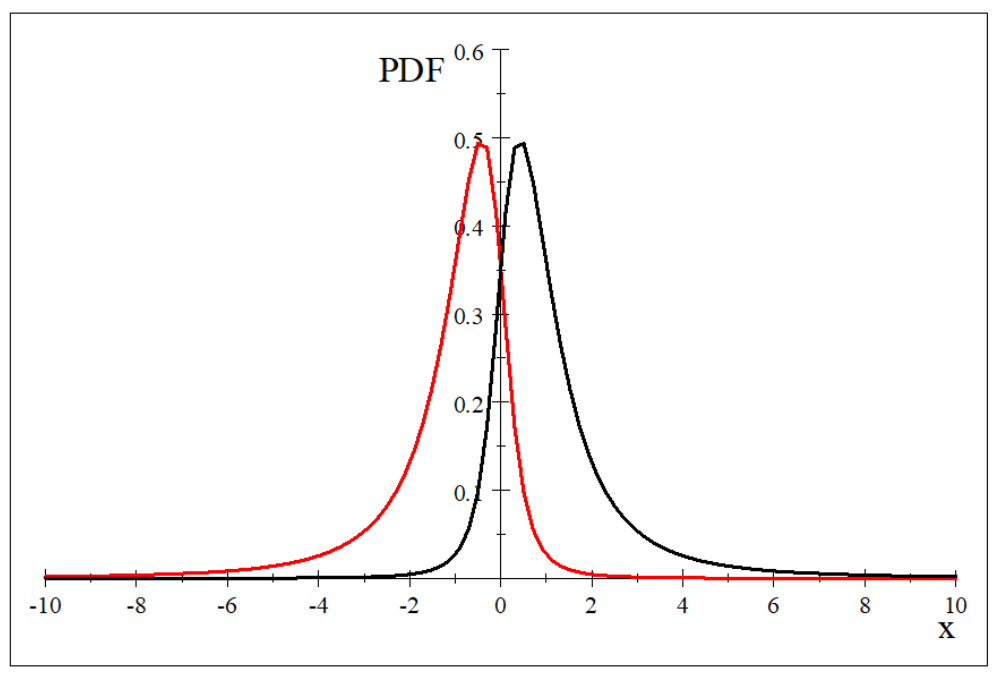

Figure 1. $f_{S T}(\mathrm{x},-2)-\operatorname{Red}, \mathrm{f}_{\mathrm{ST}}(\mathrm{x}, 2)-$ Black.

The two graphs are similar in shape. One is shifted to the right of the other. The percentile points of $f_{S T}(x,-\alpha)$ can easily be obtained from the percentile points of $f_{S T}(x, \alpha)$. Table 1 gives the percentile points of $f_{S T}(x, \alpha)$ for $\alpha=0,0.1,0.2,5,125,500$ and $\infty$.

Table 1. percentile points of $f_{S T}(x, \alpha)$.

\begin{tabular}{|l|cccccccc|}
\hline$p$ & \multicolumn{7}{|c|}{$\alpha$} \\
\cline { 2 - 9 } & 0 & 0.1 & 0.2 & 1 & 5 & 125 & 500 & $\infty$ \\
\hline 0.1 & -1.8856 & -1.7325 & -1.5764 & -0.6067 & 0.0883 & 0.1421 & 0.1421 & 0.1421 \\
0.2 & -1.0607 & -0.94656 & -0.8317 & -1.2730 & 0.2674 & 0.2887 & 0.2887 & 0.2887 \\
0.3 & -0.6172 & -0.51825 & -0.4199 & -0.8048 & 0.4334 & 0.4448 & 0.4448 & 0.4448 \\
0.4 & -0.2887 & -0.19669 & -0.1064 & 0.3783 & 0.6101 & 0.6172 & 0.6172 & 0.6172 \\
0.5 & 0 & 0.0896 & 0.1763 & 0.6211 & 0.8115 & 0.8165 & 0.8165 & 0.8165 \\
0.6 & 0.2887 & 0.37950 & 0.46639 & 0.8921 & 1.0568 & 1.0607 & 1.0607 & 1.0607 \\
0.7 & 0.6172 & 0.71369 & 0.8048 & 1.2322 & 1.3830 & 1.3862 & 1.3862 & 1.3862 \\
0.8 & 1.0697 & 1.1705 & 1.2730 & 1.7334 & 1.8827 & 1.8856 & 1.8858 & 1.8858 \\
0.9 & 1.8856 & 2.0312 & 2.1652 & 2.7433 & 2.9168 & 2.9200 & 2.9200 & 2.9200 \\
\hline
\end{tabular}

From the table it is evident that the percentile points remain the same for large positive $\alpha$ because $\frac{1}{2}-\frac{1}{\pi} \arctan$ $\alpha$ is close to zero for large $\alpha$ and the cdf as given in (2) approximate the t-distribution truncated at 0 for positive $x$. The percentile points of $f_{S T}(x,-\alpha)$ can be obtained from the above table. For example for $p=0.2$ and $\alpha=-5$, the percentile points of $f_{S T}(x,-5)$ will 1.8827 , the one corresponding to $p=0.8$ and $\alpha=5$ in $f_{S T}(x, 5)$. 
It can be shown that for the distribution given in (2) for $\alpha>0$,

$$
\lim _{n \rightarrow \infty} \frac{F^{-1}\left(1-\frac{1}{n}\right)-F^{-1}\left(1-\frac{2}{n}\right)}{F^{-1}\left(1-\frac{2}{n}\right)-F^{-1}\left(1-\frac{4}{n}\right)}=2^{1 / 2}
$$

and for $\alpha<0$,

$$
\lim _{n \rightarrow \infty} \frac{F^{-1}\left(\frac{1}{n}\right)-F^{-1}\left(\frac{2}{n}\right)}{F^{-1}\left(\frac{2}{n}\right)-F^{-1}\left(\frac{4}{n}\right)}=2^{1 / 2} .
$$

Then it follows from Ahsanullah and Nevzorov [2] that

$$
\lim _{n \rightarrow \infty} \mathrm{P}\left(X_{n, n}<a_{n}+b_{n} x\right)=e^{-x^{-2}}, \quad x \geq 0 .
$$

where $a_{n}=0$ and $F^{-1}\left(1-\frac{1}{n}\right) \approx n^{\frac{1}{2}}$, and for $\alpha<0$

$$
\begin{aligned}
\lim _{n \rightarrow \infty} \mathrm{P}\left(X_{1, n}<c_{n}+d_{n} x\right) & =1-e^{-(-x)^{-2}}, & & \mathrm{x}<0, \\
& =1, & & \mathrm{x} \geq 0,
\end{aligned}
$$

where $\mathrm{c}_{\mathrm{n}}=0$ and $d_{n}=\left|F^{-1}\left(\frac{1}{n}\right)\right| \approx n^{1 / 2}$

If $\alpha \rightarrow \infty$, then $f_{S T}(x, \alpha)$ becomes a truncated t distribution at $x=0$. In that case

$$
\begin{gathered}
\lim _{n \rightarrow \infty} \frac{F^{-1}\left(1-\frac{1}{n}\right)-F^{-1}\left(1-\frac{2}{n}\right)}{F^{-1}\left(1-\frac{2}{n}\right)-F^{-1}\left(1-\frac{4}{n}\right)}=2^{1 / 2}, \\
\lim _{n \rightarrow \infty} \mathrm{P}\left(X_{n, n}<a_{n}+b_{n} \mathrm{x}\right)=e^{-x^{-2}}, \quad x \geq 0 .
\end{gathered}
$$

with $a_{n}=0$ and $F^{-1}\left(1-\frac{1}{n}\right) \approx n^{\frac{1}{2}}$ but

$$
\lim _{n \rightarrow \infty} \frac{F^{-1}\left(\frac{1}{n}\right)-F^{-1}\left(\frac{2}{n}\right)}{F^{-1}\left(\frac{2}{n}\right)-F^{-1}\left(\frac{4}{n}\right)}=2^{-1}
$$

and

$$
\begin{aligned}
\lim _{n \rightarrow \infty} \mathrm{P}\left(X_{1, n}<c_{n}^{*}+d_{n}^{*} \mathrm{x}\right) & =1-e^{-(-x)^{-1}}, & & x<0, \\
& =1, & & x \geq 0,
\end{aligned}
$$

where $c_{n}^{*}$ and $d_{n}^{*}$ are the normalizing constants and

$$
c_{n}^{*}=0 \text { and } d_{n}^{*}=\sqrt{\frac{2}{n}} .
$$

For $\alpha=0$, we will have the t-distribution with 2 degrees of freedom. In that case

$$
\lim _{n \rightarrow \infty} \mathrm{P}\left(X_{n, n}<a_{n}+b_{n} x\right)=e^{-x^{-2}}, \quad x \geq 0 .
$$

with $a_{n}=0$ and $b_{n}=\sqrt{ }\left(\frac{n}{2}\right)$ and 


$$
\begin{aligned}
\lim _{n \rightarrow \infty} \mathrm{P}\left(X_{1, n}<c_{n}+d_{n} x\right) & =1-e^{-(-x)^{-2},}, & & x<0, \\
& =1, & & x \geq 0,
\end{aligned}
$$

where $\mathrm{c}_{\mathrm{n}}=0$ and $d_{n}=\left|F^{-1}\left(\frac{1}{n}\right)\right|=\sqrt{ }\left(\frac{n}{2}\right)$.

The following two Lemmas will be used for the characterization theorems.

Lemma 1. Suppose that $X$ is an absolutely continuous random variable with cdf $F(x)$ with $p d f f(x)$. We assume $f(x)$ is differentiable. For a continuous function $g(x)$ on $-\infty<x<\infty$ with finite $E(g(x))$ if $E(g(X) \mid X$ $\leq x)=h_{1}(x) \imath(x)$, where $h_{1}(x)$ is a differential function for all $x .-\infty<x<\infty$ and $\tau(x)=\frac{f(x)}{F(x)}$, then $f(x)=c$ $\exp \left(\int \frac{g(x)-h_{1}^{\prime}(x)}{h_{1}(x)} d x\right)$ and $c$ is determined by the condition $\int_{-\infty}^{\infty} f(x) d x=1$.

Proof. We have

$$
h_{1}(x)=\frac{\int_{0}^{x} g(u) f(u) d u}{f(x)}
$$

and

$$
\int_{0}^{x} g(u) f(u) d u=f(x) h_{1}(x) .
$$

Differentiating the above expression, we obtain

$$
g(x) f(x)=f(x) h_{1}{ }^{\prime}(x)+f^{\prime}(x) h_{1}(x)
$$

On simplification, we have

$$
\frac{f^{\prime}(x)}{f(x)}=\frac{g(x)-h_{1}^{\prime}(x)}{h_{1}(x)}
$$

Integrating both sides of the above equation, we obtain

$$
f(x)=\mathrm{c} \exp \left(\int \frac{g(x)-h_{1}^{\prime}(x)}{h_{1}(x)} d x\right)
$$

and $\mathrm{c}$ is determined by the condition $\int_{-\infty}^{\infty} f(x) d x=1$.

Lemma 2. Suppose that $X$ is an absolutely continuous random variable with $c d f F(x) p d f f(x)$. We assume $f(x)$ is differentiable. For a continuous function $g(X)$ with finite $E(g(X))$ if $E(g(X) \mid X \geq x)=h_{2}(x) r(x)$, where $h_{2}(x)$ is a differential function in $x,-\infty<x<\infty$ and $r(x)$ is the hazard function $=\frac{f(x)}{1-F(x)}$, then

$$
f(x)=c e^{-\int \frac{g(x)+h_{2}^{\prime}(x)}{h_{2}(x)} d x}
$$

and $c$ is determined by the condition $\int_{-\infty}^{\infty} f(x) d x=1$.

Proof. We have

and

$$
h_{2}(x)=\frac{\int_{x}^{\infty} g(u) f(u) d u}{f(x)}
$$

$$
\int_{x}^{\infty} g(u) f(u) d u=f(x) h_{2}(x)
$$


Differentiating the above expression, we obtain

On simplification, we have

$$
-g(x) f(x)=f(x) h_{2}^{\prime}(x)+f^{\prime}(x) h_{2}(x) .
$$

$$
\frac{f^{\prime}(x)}{f(x)}=-\frac{g(x)+h_{2}^{\prime}(x)}{h_{2}(x)}
$$

Integrating both sides of the above equation with respect to $\mathrm{x}$, we obtain

$$
f(x)=c e^{-\int \frac{g(x)+h_{2}^{\prime}(x)}{h_{2}(x)} d x}
$$

and $\mathrm{c}$ is determined by the condition $\int_{-\infty}^{\infty} f(x) d x=1$. It is easy to show that variance of the skew $\mathrm{t}$ distribution with the pdf as given in (3) does not exist but $E(X)=\frac{\alpha \sqrt{2}}{\sqrt{\left(1+\alpha^{2}\right)}}$. We use the characterization theorems using truncated first moment.

Theorem 1. Suppose that $X$ is an absolutely continuous random variable with cdf $F(x)$ with $p d f f(x)$. We assume that $f^{\prime}(x)$ exists for all $x,-\infty<x<\infty$ and $E(X)$ exists. Then $E(X \mid X \leq x)=g(x) \tau(x)$ where

and

$$
l(x)=\frac{f(x)}{F(x)}
$$

$$
\begin{gathered}
g(x)=\frac{\left(x^{2}+2\right)^{\frac{3}{2}} p(x)}{1+\frac{2}{\pi} \arctan \frac{\alpha x}{\sqrt{x^{2}+2}}+\frac{2}{\pi} \frac{\alpha x \sqrt{x^{2}+2}}{\left(1+\alpha^{2}\right) x^{2}+2}} \\
p(x)=\frac{-\alpha}{\sqrt{2\left(1+\alpha^{2}\right)}}+\frac{1}{\sqrt{2\left(1+\alpha^{2}\right)}} \arctan \left(\sqrt{\frac{1+\alpha^{2}}{2}} x\right)-\frac{1}{\sqrt{x^{2}+2}}-\frac{1}{\sqrt{x^{2}+2}}\left(\frac{2}{\pi} \arctan \frac{\alpha x}{\sqrt{x^{2}+2}}\right)
\end{gathered}
$$

if and only if

$$
f(x)=\frac{1}{\left(x^{2}+2\right)^{\frac{3}{2}}}\left(1+\frac{2}{\pi} \arctan \frac{\alpha x}{\sqrt{x^{2}+2}}+\frac{2}{\pi} \frac{\alpha x \sqrt{x^{2}+2}}{\left(1+\alpha^{2}\right) x^{2}+2}\right) .
$$

Proof. We have

$$
\begin{aligned}
f(x) g(x) & =\int_{-\infty}^{x} \frac{u}{\left(u^{2}+2\right)^{\frac{3}{2}}}\left(1+\frac{2}{\pi} \arctan \frac{\alpha u}{\sqrt{u^{2}+2}}+\frac{2}{\pi} \frac{\alpha u \sqrt{u^{2}+2}}{\left(1+\alpha^{2}\right) u^{2}+2}\right) d u \\
& =\frac{-\alpha}{\sqrt{2\left(1+\alpha^{2}\right)}}+\frac{1}{\sqrt{2\left(1+\alpha^{2}\right)}} \arctan \left(\sqrt{\frac{1+\alpha^{2}}{2}} x\right)-\frac{1}{\sqrt{x^{2}+2}}-\frac{2}{\pi} \frac{1}{\sqrt{x^{2}+2}} \arctan \frac{\alpha x}{\sqrt{x^{2}+2}} . \\
& =p(x)
\end{aligned}
$$

Thus 


$$
g(x)=\frac{\left(x^{2}+2\right)^{\frac{3}{2}} p(x)}{1+\frac{2}{\pi} \arctan \frac{\alpha x}{\sqrt{x^{2}+2}}+\frac{2}{\pi} \frac{\alpha x \sqrt{x^{2}+2}}{\left(1+\alpha^{2}\right) x^{2}+2}} .
$$

Suppose

$$
g(x)=\frac{\left(x^{2}+2\right)^{\frac{3}{2}} p(x)}{1+\frac{2}{\pi} \arctan \frac{\alpha x}{\sqrt{x^{2}+2}}+\frac{2}{\pi} \frac{\alpha x \sqrt{x^{2}+2}}{\left(1+\alpha^{2}\right) x^{2}+2}+\frac{2}{\pi} \frac{\alpha x \sqrt{x^{2}+2}}{\left(1+\alpha^{2}\right) x^{2}+2}},
$$

using the relation $p^{\prime}(x)=x f(x)$ and $p(x) \frac{d}{d x}\left(\frac{1}{f(x)}\right)=-\frac{p(x)}{f(x)} \frac{d}{d x} \ln f(x)$, we have

$$
g^{\prime}(x)=x-g(x)\left(\frac{-3 x}{x^{2}+2}+\frac{\frac{4}{\pi} \frac{\alpha}{\sqrt{x^{2}+2}\left(x^{2} \alpha^{2}+x^{2}+2\right)}+\frac{2}{\pi} \frac{\alpha}{\sqrt{x^{2}+2}} \frac{-2 x^{2} \alpha^{2}+2 x^{2}+4}{\left(x^{2} \alpha^{2}+x^{2}+2\right)^{2}}}{1+\frac{2}{\pi} \arctan \frac{\alpha x}{\sqrt{x^{2}+2}}+\frac{2}{\pi} \frac{\alpha x \sqrt{x^{2}+2}}{\left(1+\alpha^{2}\right) x^{2}+2}}\right) .
$$

On simplification, we obtain

$$
\frac{x-g^{\prime}(x)}{g(x)}=\frac{-3 x}{x^{2}+2}+\frac{\frac{4}{\pi} \frac{\alpha}{\sqrt{x^{2}+2}\left(x^{2} \alpha^{2}+x^{2}+2\right)}+\frac{2}{\pi} \frac{\alpha}{\sqrt{x^{2}+2}} \frac{-2 x^{2} \alpha^{2}+2 x^{2}+4}{\left(x^{2} \alpha^{2}+x^{2}+2\right)^{2}}}{1+\frac{2}{\pi} \arctan \frac{\alpha x}{\sqrt{x^{2}+2}}+\frac{2}{\pi} \frac{\alpha x \sqrt{x^{2}+2}}{\left(1+\alpha^{2}\right) x^{2}+2}} .
$$

By Lemma 1, we have

$$
\frac{f^{\prime}(x)}{f(x)}=\frac{-3 x}{x^{2}+2}+\frac{\frac{4}{\pi} \frac{\alpha}{\sqrt{x^{2}+2}\left(x^{2} \alpha^{2}+x^{2}+2\right)}+\frac{2}{\pi} \frac{\alpha}{\sqrt{x^{2}+2}} \frac{-2 x^{2} \alpha^{2}+2 x^{2}+4}{\left(x^{2} \alpha^{2}+x^{2}+2\right)^{2}}}{1+\frac{2}{\pi} \arctan \frac{\alpha x}{\sqrt{x^{2}+2}}+\frac{2}{\pi} \frac{\alpha x \sqrt{x^{2}+2}}{\left(1+\alpha^{2}\right) x^{2}+2}} .
$$

On integrating both sides of the equation, with respect to $\mathrm{x}$, we have

$$
f(x)=c \frac{1}{\left(x^{2}+2\right)^{\frac{3}{2}}}\left(1+\frac{2}{\pi} \arctan \frac{\alpha x}{\sqrt{x^{2}+2}}+\frac{2}{\pi} \frac{\alpha x \sqrt{x^{2}+2}}{\left(1+\alpha^{2}\right) x^{2}+2}\right) .
$$

Using the boundary condition $\int_{-\infty}^{\infty} f(x) d x=1$, we have $c=1$.

We will give here a characterization of the skew-t distribution using the left truncated first moment.

Theorem 2. Suppose that $X$ is an absolutely continuous random variable with cdf $F(x)$ with $p d f f(x)$. We assume that $E(X)$ is finite and $f^{\prime}(x)$ exists for all $x,-\infty<x<\infty$. Then $E(X \mid X \geq x)=m(x) r(x)$, where 


$$
\begin{gathered}
r(x)=\frac{f(x)}{1-F(x)} \\
m(x)=\frac{n(x)}{f(x)}, \quad n(x)=\frac{\sqrt{2} \alpha}{\sqrt{\alpha^{2}+1}}-\left(\frac{-\alpha}{\sqrt{2\left(1+\alpha^{2}\right)}}+\frac{1}{\sqrt{2\left(1+\alpha^{2}\right)}} \arctan \left(\sqrt{\frac{1+\alpha^{2}}{2}} x\right)\right. \\
\left.-\frac{1}{\sqrt{x^{2}+2}}-\frac{1}{\sqrt{x^{2}+2}}\left(\frac{2}{\pi} \arctan \frac{\alpha x}{\sqrt{x^{2}+2}}\right)\right)
\end{gathered}
$$

if and only if

$$
f(x)=\frac{1}{\left(x^{2}+2\right)^{\frac{3}{2}}}\left(1+\frac{2}{\pi} \arctan \frac{\alpha x}{\sqrt{x^{2}+2}}+\frac{2}{\pi} \frac{\alpha x \sqrt{x^{2}+2}}{\left(1+\alpha^{2}\right) x^{2}+2}\right) .
$$

Proof. We have

$$
\begin{aligned}
f(x) m(x) & =\int_{x}^{\infty} \frac{u}{\left(u^{2}+2\right)^{\frac{3}{2}}}\left(1+\frac{2}{\pi} \arctan \frac{\alpha u}{\sqrt{u^{2}+2}}+\frac{2}{\pi} \frac{\alpha u \sqrt{u^{2}+2}}{\left(1+\alpha^{2}\right) u^{2}+2}\right) d u \\
& =E(X)-\int_{-\infty}^{x} \frac{u}{\left(u^{2}+2\right)^{\frac{3}{2}}}\left(1+\frac{2}{\pi} \arctan \frac{\alpha u}{\sqrt{u^{2}+2}}+\frac{2}{\pi} \frac{\alpha u \sqrt{u^{2}+2}}{\left(1+\alpha^{2}\right) u^{2}+2}\right) d u \\
& =\frac{\sqrt{2} \alpha}{\sqrt{\alpha^{2}+1}}-\left(\frac{-\alpha}{\sqrt{2\left(1+\alpha^{2}\right)}}+\frac{1}{\sqrt{2\left(1+\alpha^{2}\right)}} \arctan \left(\sqrt{\frac{1+\alpha^{2}}{2}} x\right)\right. \\
& =\frac{1}{\sqrt{x^{2}+2}}-\frac{2}{\pi} \frac{1}{\sqrt{x^{2}+2}} \arctan \frac{\alpha x}{\sqrt{x^{2}+2}} \\
& n(x) .
\end{aligned}
$$

Thus,

$$
m(x)=\frac{\left(x^{2}+2\right)^{\frac{3}{2}} n(x)}{1+\frac{2}{\pi} \arctan \frac{\alpha x}{\sqrt{x^{2}+2}}+\frac{2}{\pi} \frac{\alpha x \sqrt{x^{2}+2}}{\left(1+\alpha^{2}\right) x^{2}+2}} .
$$

Suppose

$$
m(x)=\frac{\left(x^{2}+2\right)^{\frac{3}{2}} n(x)}{1+\frac{2}{\pi} \arctan \frac{\alpha x}{\sqrt{x^{2}+2}}+\frac{2}{\pi} \frac{\alpha x \sqrt{x^{2}+2}}{\left(1+\alpha^{2}\right) x^{2}+2}},
$$

then 


$$
m^{\prime}(x)=-x-m(x)\left(\frac{-3 x}{x^{2}+2}+\frac{\frac{4}{\pi} \frac{\alpha}{\sqrt{x^{2}+2}\left(x^{2} \alpha^{2}+x^{2}+2\right)}+\frac{2}{\pi} \frac{\alpha}{\sqrt{x^{2}+2}} \frac{-2 x^{2} \alpha^{2}+2 x^{2}+4}{\left(x^{2} \alpha^{2}+x^{2}+2\right)^{2}}}{1+\frac{2}{\pi} \arctan \frac{\alpha x}{\sqrt{x^{2}+2}}+\frac{2}{\pi} \frac{\alpha x \sqrt{x^{2}+2}}{\left(1+\alpha^{2}\right) x^{2}+2}}\right) .
$$

Thus

$$
\frac{\left.x+m^{\prime} x\right)}{m(x)}=-\frac{-3 x}{x^{2}+2}+\frac{\frac{4}{\pi} \frac{\alpha}{\sqrt{x^{2}+2}\left(x^{2} \alpha^{2}+x^{2}+2\right)}+\frac{2}{\pi} \frac{\alpha}{\sqrt{x^{2}+2}} \frac{-2 x^{2} \alpha^{2}+2 x^{2}+4}{\left(x^{2} \alpha^{2}+x^{2}+2\right)^{2}}}{1+\frac{2}{\pi} \arctan \frac{\alpha x}{\sqrt{x^{2}+2}}+\frac{2}{\pi} \frac{\alpha x \sqrt{x^{2}+2}}{\left(1+\alpha^{2}\right) x^{2}+2}}
$$

By Lemma 2 we have

$$
\frac{f^{\prime}(x)}{f(x)}=\frac{-3 x}{x^{2}+2}+\frac{\frac{4}{\pi} \frac{\alpha}{\sqrt{x^{2}+2}\left(x^{2} \alpha^{2}+x^{2}+2\right)}+\frac{2}{\pi} \frac{\alpha}{\sqrt{x^{2}+2}} \frac{-2 x^{2} \alpha^{2}+2 x^{2}+4}{\left(x^{2} \alpha^{2}+x^{2}+2\right)^{2}}}{1+\frac{2}{\pi} \arctan \frac{\alpha x}{\sqrt{x^{2}+2}}+\frac{2}{\pi} \frac{\alpha x \sqrt{x^{2}+2}}{\left(1+\alpha^{2}\right) x^{2}+2}} .
$$

Integrating the both sides of the above equation with respect to $x$, we obtain

$$
f(x)=c \frac{1}{\left(x^{2}+2\right)^{\frac{3}{2}}}\left(1+\frac{2}{\pi} \arctan \frac{\alpha x}{\sqrt{x^{2}+2}}+\frac{2}{\pi} \frac{\alpha x \sqrt{x^{2}+2}}{\left(1+\alpha^{2}\right) x^{2}+2}\right) .
$$

Using the boundary condition $\int_{-\infty}^{\infty} f(x) d x=1$, we have $c=1$.

\section{References}

[1] Ahsanullah, M., Kibria, B.M.G. and M. Shakil. (2001). Normal and Student's t-distributions and Their Applications. AtlantisPress, Paris, France.

[2] Ahsanullah, M., and Nevzorov, V. B.(2001). Ordered random variables. Nova Science Publishers, New York,NY, USA.

[3] Ahsanullah, M., Shakil, M. and Kibria, B.M.G. (2005). Characterizations of folded t-distribution. Journal of Statistical Distributions and Applications, 3, 1-15.'

[4] Arellano-Valle,R.B. and Genton, M.G. (2005). On fundamental skew distributions, J. Multiv. Ana. 96, 2005, 93-116.

[5] Arnold, B.C. and Lin, G.D. (2004). Characterizations of skew normal and generalized chi-square distributions. Sankhya, 66 , 593-606.

[6] Azzalini, A. (2014). The Skew - Normal and Related Families. Cambridge University Press.

[7] Azzalini, A. and Capitanio, A. (2003). Distributions generated by perturbation of symmetry with emphasis on a multivariate skew t distribution. J.R. Statist. Soc. series B.65, 367-389.

[8] Azzalini, A. (1985).A class of distributions which include the normal ones. Skand. J. Statist. 12, 171-178.

[9] Jamalizadeh, A., Khosravi and Balakrishnan, N. (2009). Recurrence relations of for distributions of a skew $t$ and a linear combination of order statistics from bivariate t. Computational Statistics and Data Analysis 53, 2009, 847-852.

[10] Nevzorov, V.B., Balakrishnan, N. and Ahsanullah, M. (2001). Simple characterization of Student-t distribution. Statistician 53(3), 395-400.

[11] Yanev, G.P. and Ahsanullah, M. (2011). Characterization of Student-t distribution via regression of order statistics. Statistician, $1-7$. 